\title{
Accuracy and Precision of Plane Wave Vector Flow Imaging for Laminar and Complex Flow In Vivo
}

Jensen, Jonas; Traberg, Marie Sand; Villagómez Hoyos, Carlos Armando; Olesen, Jacob Bjerring; Tomov, Borislav Gueorguiev; Moshavegh, Ramin; Stuart, Matthias Bo; Ewertsen, Caroline; Hansen, Kristoffer Lindskov; Bachmann Nielsen, Michael

Total number of authors:

11

Published in:

Proceedings of IEEE International Ultrasonics Symposium

Publication date:

2017

Document Version

Peer reviewed version

Link back to DTU Orbit

Citation (APA):

Jensen, J., Traberg, M. S., Villagómez Hoyos, C. A., Olesen, J. B., Tomov, B. G., Moshavegh, R., Stuart, M. B., Ewertsen, C., Hansen, K. L., Bachmann Nielsen, M., \& Jensen, J. A. (2017). Accuracy and Precision of Plane Wave Vector Flow Imaging for Laminar and Complex Flow In Vivo. In Proceedings of IEEE International Ultrasonics Symposium IEEE.

\section{General rights}

Copyright and moral rights for the publications made accessible in the public portal are retained by the authors and/or other copyright owners and it is a condition of accessing publications that users recognise and abide by the legal requirements associated with these rights.

- Users may download and print one copy of any publication from the public portal for the purpose of private study or research.

- You may not further distribute the material or use it for any profit-making activity or commercial gain

- You may freely distribute the URL identifying the publication in the public portal 


\title{
Accuracy and Precision of Plane Wave Vector Flow Imaging for Laminar and Complex Flow In Vivo
}

\author{
Jonas Jensen ${ }^{1,2}$, Marie Sand Traberg ${ }^{1}$, Carlos Armando Villagómez Hoyos ${ }^{1}$, Jacob Bjerring Olesen ${ }^{1,3}$, \\ Borislav Tomov ${ }^{1}$, Ramin Moshavegh ${ }^{1,3}$, Matthias Bo Stuart ${ }^{1}$, Caroline Ewertsen ${ }^{4}$, Kristoffer Lindskov Hansen ${ }^{4}$, \\ Michael Bachmann Nielsen ${ }^{4}$, and Jørgen Arendt Jensen ${ }^{1}$ \\ ${ }^{1}$ Center for Fast Ultrasound Imaging, Department of Electrical Engineering, \\ Technical University of Denmark, DK-2800 Lyngby, Denmark \\ ${ }^{2}$ Department of Micro- and Nanotechnology, Technical University of Denmark, DK-2800 Lyngby, Denmark \\ ${ }^{3}$ BK Ultrasound, DK-2730 Herlev, Denmark \\ ${ }^{4}$ Department of Radiology, Copenhagen University Hospital, DK-2100 Copenhagen, Denmark
}

\begin{abstract}
In this study, a comparison between velocity fields for a plane wave 2-D vector flow imaging (VFI) method and a computational fluid dynamics (CFD) simulation is made. VFI estimates are obtained from the scan of a flow phantom, which mimics the complex flow conditions in the carotid artery. Furthermore, the precision of the VFI method is investigated under laminar and complex flow conditions in vivo. The carotid bifurcation of a healthy volunteer was scanned using both fast plane wave ultrasound and magnetic resonance imaging (MRI). The acquired MRI geometry of the bifurcation was used for fabricating an anthropomorphic flow phantom, which was also ultrasound scanned. The same geometry was used in a CFD simulation to calculate the velocity field. Results showed that similar flow patterns and vortices were estimated using CFD and VFI in the phantom. Velocity magnitudes were estimated with a mean difference within $15 \%$, however, it was $23 \%$ in the external branch. For the in vivo scan, the precision in terms of mean standard deviation (SD) of estimates aligned to the cardiac cycle was highest in the center of the common carotid artery (SD $4.7^{\circ}$ for angles) and lowest in the external branch and close to the vessel wall (SD $15.0^{\circ}$ for angles).
\end{abstract}

\section{INTRODUCTION}

The combination of high-frame rate ultrasound imaging methods and 2-D vector flow imaging (VFI) [1]-[4] presents several advantages compared to conventional velocity imaging: Rapid blood flow patterns can be followed at hundreds or thousands of frames per second, the velocity estimates are angle-independent, and quantitative estimates are continuously available everywhere in the image. This enables visualization of complex blood flow patterns, e.g. the formation of vortices, which often form around bifurcations and valves. The VFI methods must provide precise and accurate velocity estimates for both laminar and complex flow when measured in vivo. However, when investigating the performance of a VFI method in vivo, two challenges arise: (i) independent methods such as spectral Doppler and magnetic resonance angiography (MRA) do not have sufficient spatial and temporal resolution to accurately capture complex flow patterns in vivo, which makes a comparison to VFI difficult; and (ii) data should be acquired for at least two to three heart beats to evaluate the precision in terms of repeatability of VFI estimates, but this generates more transducer element data than most scanners can store.

In relation to (i), an alternative approach to investigate complex flow is to perform a realistic phantom study. Using an anthropomorphic geometry for the phantom fabrication, complex flow patterns may be measured in a realistic vessel geometry. The same geometry and boundary conditions can be used in a simulation of the velocity field using computational fluid dynamics (CFD). The CFD simulated velocities can then be compared to the VFI estimates obtained from the phantom. In relation to (ii), all element data can be stored in scans for up to $10 \mathrm{~s}$ by using the experimental scanner SARUS [5], which enables evaluation of the precision of the estimates from several heart beats.

The method for VFI in this paper was based on plane wave imaging and a combination of transverse oscillation (TO) and directional beamforming (DB), which was previously validated in simulations [6]. The objective of this paper was to investigate the accuracy and precision of the method under laminar and complex flow conditions in the carotid bifurcation of a healthy volunteer.

The accuracy of the method was investigated by comparing ultrasound VFI estimates obtained from an anthropomorphic phantom measurement to a CFD simulated velocity field derived from the same geometry. The precision was investigated in vivo by calculating the repeatability of the VFI estimates at several estimation points in the bifurcation.

\section{Methods}

A 27 year old healthy volunteer participated after informed consent, and the study was approved by the National Committee on Biomedical Research Ethics (Protocol No. H-12014-fsp-072). An in vivo VFI scan was conducted during a session in the morning, and an MRI scan was performed in the evening.

\section{A. In vivo VFI scan}

A plane wave VFI scan was performed using a linear array transducer (BK 8L2, BK Ultrasound, Herlev, Denmark), which was connected to the SARUS scanner [5]. The carotid bulb was 
scanned with the best possible view of the bifurcation, and a duplex sequence consisting of flow and B-mode emissions was employed (4.1 MHz center frequency, $10 \mathrm{kHz}$ PRF, plane waves at $\left.-15^{\circ}, 0^{\circ}, 15^{\circ}\right)$ [6]. Element $\mathrm{RF}$ data were acquired for a total of $10 \mathrm{~s}$. Echo-canceling of beamformed data was performed using an energy-based filter with a manual threshold [7].

A two-step procedure was employed for velocity estimation: First, the TO method was used to estimate an initial flow angle $\theta$, which was calculated from the axial and lateral velocities $v_{z}$ and $v_{x}$, respectively. Second, three DB lines were focused around $\theta$ at each estimation point to find a refined angle $\hat{\theta}$ using the largest normalized cross-correlation estimate [6]. The velocity magnitude was estimated along the direction $\hat{\theta}$ using a cross-correlation estimator.

\section{B. MRI scan}

A $1.5 \mathrm{~T}$ whole body scanner (Magnetom Avanto, Siemens, Erlangen, Germany) was used with a head and a neck matrix coil. The carotid artery was MRI scanned to acquire anatomical images of the common carotid artery (CCA) and carotid bifurcation. The repetition time of the sequence was $42 \mathrm{~ms}$, echo-time $3 \mathrm{~ms}$, pixel resolution $1.1 \times 1.1 \mathrm{~mm}$, and slice thickness $5 \mathrm{~mm}$. The anatomical images were used to create a 3-D geometry representing the carotid artery of the volunteer. A CFD model and a flow phantom were produced from the 3-D geometry as described in the following sections.

\section{Flow phantom fabrication and scan}

An anthropomorphic flow phantom matching the flow domain of the original scanned vessel was fabricated using stereolithography [8]. After 3-D printing of the vessel core, it was fixated in a container and cast in polyvinyl alcohol (PVC) cryogel to obtain a tissue-mimicking surrounding medium. The elastic properties of the cast material were controlled by varying the number of freeze-thaw cycles. After completion of three cycles, the core was removed manually to obtain the wall-less phantom.

A flow system (CompuFlow 1000, Shelley Medical Imaging Technologies, Toronto, Canada) was connected to the phantom and circulated a blood-mimicking fluid, which had a viscosity of $4.1 \cdot 10^{-3} \mathrm{~Pa} \cdot \mathrm{s}$ and a density of $1030 \mathrm{~kg} / \mathrm{m}^{3}$. The CompuFlow system was set to generate a standard carotid artery waveform. The transducer was placed on the phantom to scan the bifurcation. The SARUS scanner was used for data acquisition with the same sequence and parameters as for the in vivo scan.

\section{CFD model}

The flow simulation in the CFD model was governed by solving the Navier-Stokes equations. The properties of the emulated fluid matched those of the blood-mimicking fluid used for the phantom study. It was assumed that the fluid was Newtonian, entrance effects were discarded, the vessel was rigid, and the flow had reached a steady state of pulsation.
The inlet condition was constructed using the WomersleyEvans model [9], [10]. In details, the individual components of the mean spatial velocity curve, which was obtained from the measured plane wave VFI data at a point in the CCA, were found by Fourier decomposition. Thereafter, superposition of the first ten harmonics provided a smooth spatial velocity variation. This result was then used to reconstruct the velocity profile in both time and space using the Womersley-Evans model. An outlet pressure of $0 \mathrm{~Pa}$ was set for the two exit branches, while a no-slip condition with $\vec{v}=0 \mathrm{~m} / \mathrm{s}$ was set at the walls of the flow domain. The CFD simulation was carried out in Comsol Multiphysics (v5.2a, Comsol AB, Stockholm, Sweden).

\section{E. Evaluation}

Prior to the comparison between the flow field of the CFD simulation and the phantom VFI scan, the geometries were aligned. Rotation and translation of the geometries were performed manually after visual inspection. A quantitative comparison of velocities acquired from CFD and VFI were made at selected planes in the bifurcation. Furthermore, the vorticity was calculated using the rotational frequency:

$$
\omega=\nabla \times \vec{v}=\left(\frac{\partial v_{z}}{\partial x}-\frac{\partial v_{x}}{\partial z}\right),
$$

where $\nabla$ is the nabla operator. The mean rotational frequency was calculated for an area covering a (part of a) vortex.

For the precision study, a medical doctor evaluated the in vivo scan by selecting evaluation points in the center of the CCA, close to the vessel wall of CCA, in the internal carotid artery (ICA), and in the external (ECA). An automatic procedure calculated the mean cardiac period and coherently aligned the velocity magnitude and angle estimates according to the cardiac period. The standard deviation $\sigma(t)$ at time $t$ was calculated among the aligned estimates. The mean standard deviation (SD) was

$$
S D=\sqrt{\frac{1}{N_{f}} \sum_{t=1}^{N_{f}} \sigma(t)^{2}},
$$

where $N_{f}$ is the number of frames encompassing a cardiac cycle. The mean SD of velocity magnitude estimates, (2) was calculated relative to the peak velocity magnitude $v_{0}$.

\section{RESULTS}

\section{A. Comparison between phantom VFI and CFD simulation}

A frame from the comparison between CFD simulation and the VFI scan is shown in Fig. 1. The frame is within systolic deceleration (0.32 s) and shows similar flow patterns for CFD and VFI. Both the CFD and VFI showed a large vortex or recirculation zone, which extended from the ICA into the CCA. There were small differences in the extend of the recirculation zone and velocity magnitudes, but similar flow patterns were provided. The jet of streamlined forward flow found close to the inner vessel wall of the ICA in the CFD image was also measured using VFI. A small vortex in 

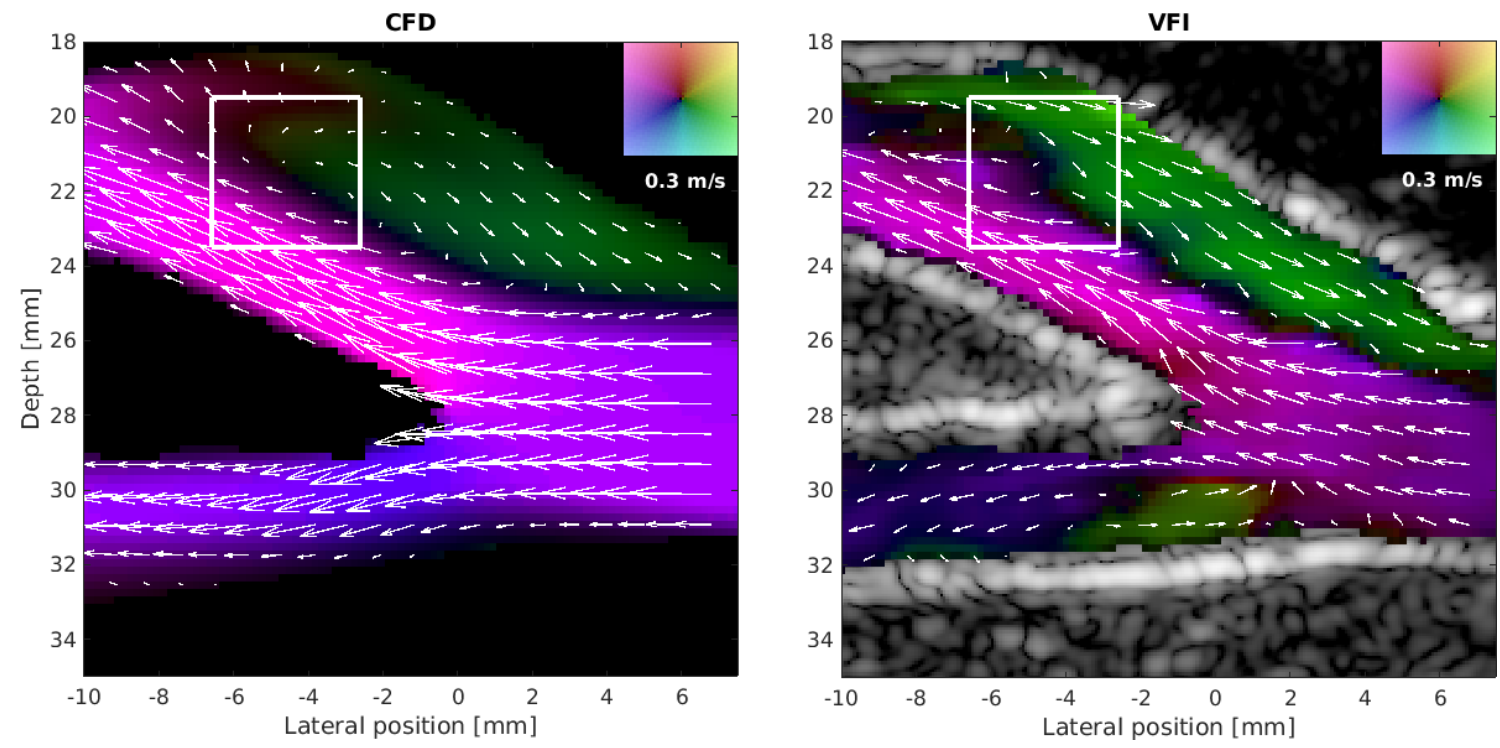

Fig. 1. Velocity estimates from CFD simulation (left image) and VFI scan of phantom (right image). The frame is within the systolic deceleration $(t=$ $0.32 \mathrm{~s}$ ). The vorticity within the white box covering a part of the recirculation zone is shown in Fig. 3 .
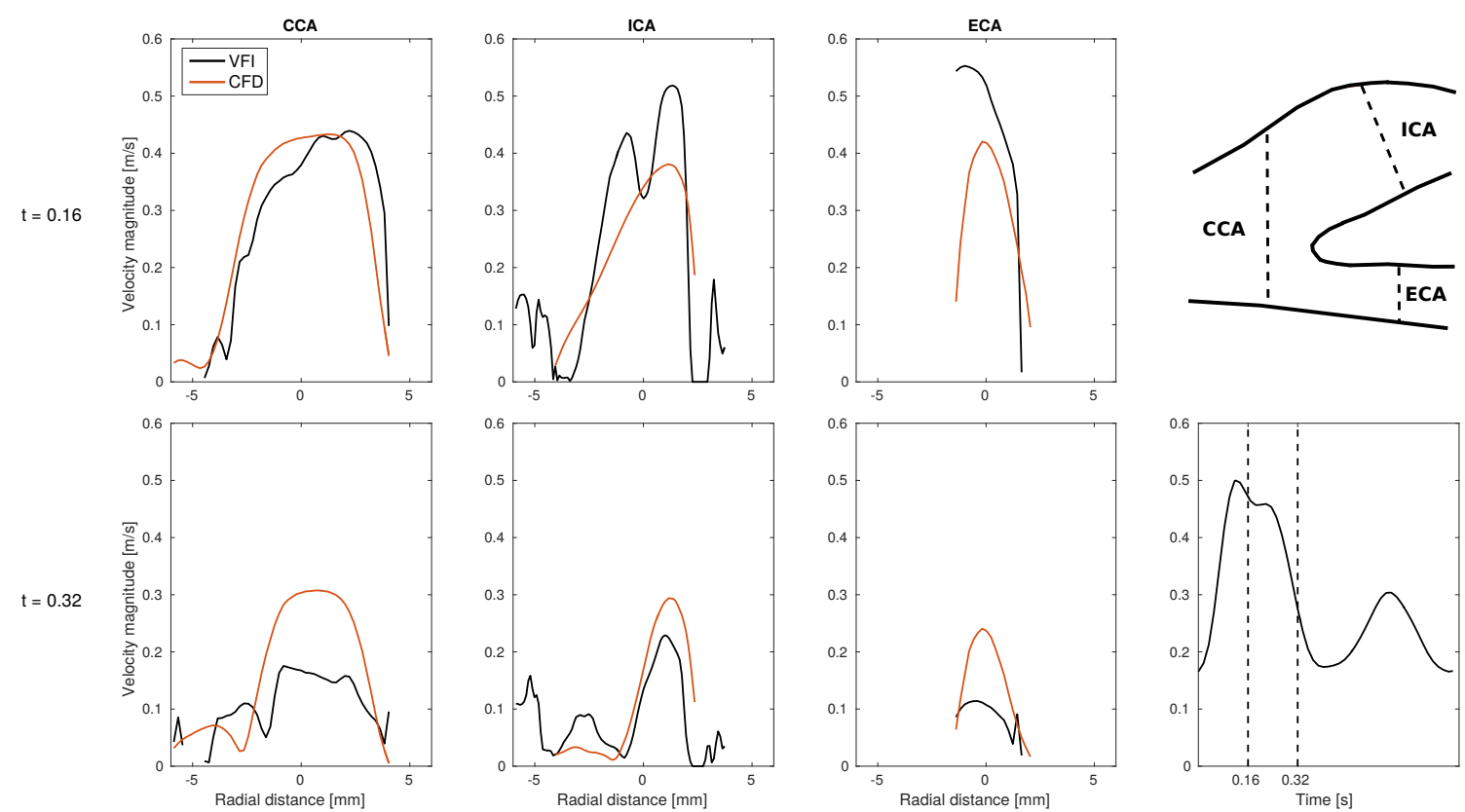

Fig. 2. Comparison of velocity magnitude profiles at selected locations in the CCA (left column), ICA (middle column), and ECA (right column). Sampling was performed at $t=0.16 \mathrm{~s}$ (top row), $t=0.32 \mathrm{~s}$ (bottom row). Red profiles indicate CFD estimates and black VFI estimates.

the ECA was measured using VFI as indicated by the green area in the lower branch in Fig. 1 (right). However, this vortex was not visible in the CFD.

A more quantitative comparison between CFD and VFI is shown in Fig. 2, where the velocity magnitude profiles at selected locations in the CCA, ICA, and ECA are shown at peak systole $(t=0.16 \mathrm{~s})$ and systolic deceleration $(t=0.32 \mathrm{~s})$.

The mean difference between CFD and VFI was of $-1.4 \%$ for the velocity profile in the CCA at peak systole, while it was $23.6 \%$ for the ECA. During systolic deceleration (Fig. 2 bottom row), lower velocities were estimated in both the CCA and ECA (mean difference was $-12.3 \%$ and $-13.3 \%$, respectively), when using VFI compared to CFD. However, the velocity magnitudes of the backflow in the ICA from $-5 \mathrm{~mm}$ to $0 \mathrm{~mm}$ were larger for VFI, which is also shown in Fig. 1 .

The rotation of flow in the recirculation zone inside the white box in Fig. 1 was quantified by calculating the mean rotational frequency as a function of time, and the result is shown in Fig. 3. The two peaks of the rotational frequency for VFI at $0.14 \mathrm{~s}$ and $0.3 \mathrm{~s}$ revealed that a vortex or recirculation evolved at two time instances, while a single recirculation built up after $0.15 \mathrm{~s}$ in the CFD simulation and lasted until $0.3 \mathrm{~s}$. For the remaining cardiac period (from $0.3 \mathrm{~s}$ to $0.84 \mathrm{~s}$ ) there is a good agreement between CFD and VFI for the rotational 


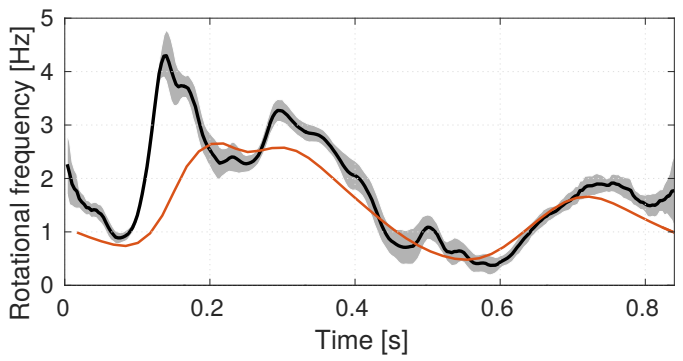

Fig. 3. The rotational frequency as a function of time for the recirculation zone inside the box, which is marked in Fig. 1. Black graph is the mean VFI estimates, gray area is the SD of VFI estimates, and red graph is CFD estimates.

frequency.

\section{B. Precision in vivo}

The precision of velocity magnitude and angle estimates was calculated for evaluation points in the center of the CCA, close to the vessel wall of the CCA, in the ICA, and the ECA. The points are indicated on the VFI frame shown in Fig. 4. The highest precision was obtained in the center of the CCA (3.0\% for velocities and $4.7^{\circ}$ for angles) and in the ICA $\left(5.5 \%\right.$ and $\left.6.5^{\circ}\right)$, where the highest velocities were estimated and flow was mainly unidirectional. Slightly lower precisions were obtained in the $\operatorname{ECA}\left(5.1 \%\right.$ and $\left.13.3^{\circ}\right)$ and close to the vessel wall of the CCA $\left(7.3 \%\right.$ and $\left.15.0^{\circ}\right)$. Lower velocities and a larger variation in the angles were found for these evaluation points.

The rotational frequency was calculated for the vortex indicated by the white square in Fig. 4 and for all frames during the $10 \mathrm{~s}$ scan. After alignment of estimates to the cardiac cycle, the mean SD of the rotational frequency was calculated to $6.2 \%$. This indicated a high cardiac cycle-tocycle repeatablity of the velocity estimates and flow patterns in a zone covering the vortex.

\section{Discussion AND CONCLUSION}

A comparison between flow in a carotid bifurcation phantom using VFI and simulated CFD velocities was made. Similar flow patterns were obtained, and the mean difference between VFI and CFD was within $23 \%$ for velocities. However, the alignment of the transducer scan plane in the phantom and the CFD geometry was not perfect, and the assumptions of rigid walls and inlet and outlet boundary conditions may represent limitations in the CFD model.

The study showed that the precision in vivo was highest in the center of the common carotid artery and lowest in the external branch (standard deviation for angles $4.7^{\circ}$ and $15.0^{\circ}$ ). This indicated that quantitative velocity estimates could be obtained in the carotid artery of the healthy volunteer.

\section{ACKNOWLEDGMENTS}

This work was supported by grant 82-2012-4 from the Danish National Advanced Technology Foundation and by BK Ultrasound Aps.

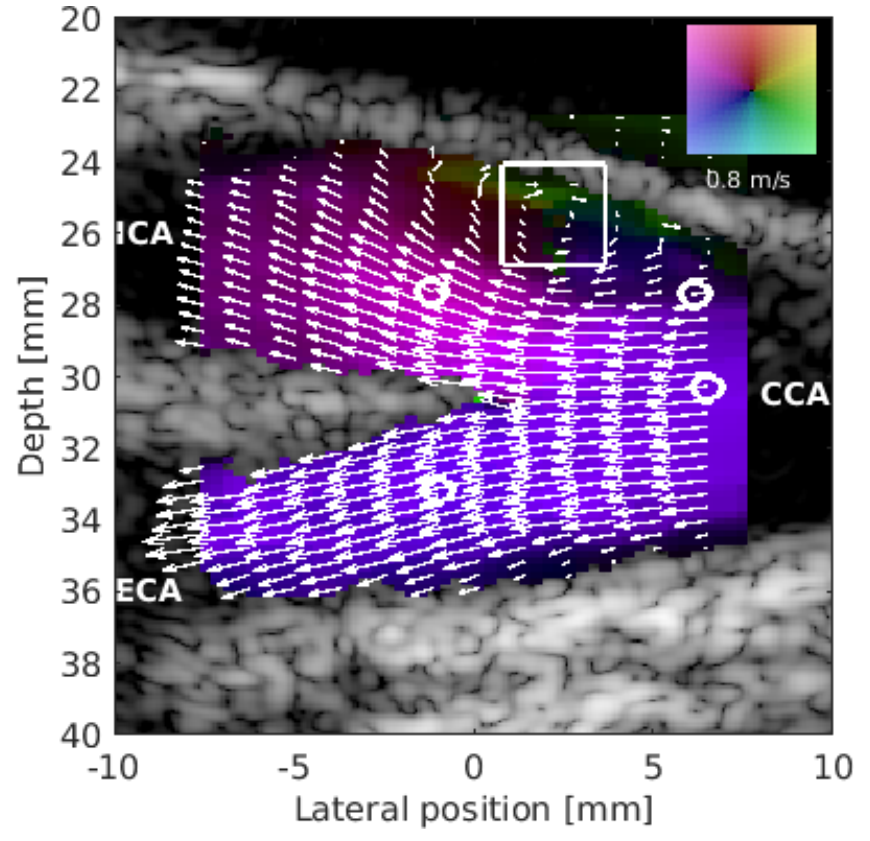

Fig. 4. VFI frame from the in vivo scan of the carotid bifurcation of the volunteer. The frame is from peak systole, and the white circles indicate the evaluation points selected by the medical doctor. The white box indicate the area, where the rotational frequency of the vortex was calculated.

\section{REFERENCES}

[1] S. I. Nikolov and J. A. Jensen, "In-vivo synthetic aperture flow imaging in medical ultrasound," IEEE Trans. Ultrason., Ferroelec., Freq. Contr., vol. 50, no. 7, pp. 848-856, 2003.

[2] J. Udesen, F. Gran, K. L. Hansen, J. A. Jensen, C. Thomsen, and M. B. Nielsen, "High frame-rate blood vector velocity imaging using plane waves: simulations and preliminary experiments," IEEE Trans. Ultrason., Ferroelec., Freq. Contr., vol. 55, no. 8, pp. 1729-1743, 2008.

[3] J. Bercoff, G. Montaldo, T. Loupas, D. Savery, F. Meziere, M. Fink, and M. Tanter, "Ultrafast compound Doppler imaging: providing full blood flow characterization," IEEE Trans. Ultrason., Ferroelec., Freq. Contr., vol. 58, no. 1, pp. 134-147, January 2011.

[4] I. K. Ekroll, A. Swillens, P. Segers, T. Dahl, H. Torp, and L. Lovstakken, "Simultaneous quantification of flow and tissue velocities based on multi-angle plane wave imaging," IEEE Trans. Ultrason., Ferroelec., Freq. Contr., vol. 60, no. 4, pp. 727-738, 2013.

[5] J. A. Jensen, H. Holten-Lund, R. T. Nilsson, M. Hansen, U. D. Larsen, R. P. Domsten, B. G. Tomov, M. B. Stuart, S. I. Nikolov, M. J. Pihl, Y. Du, J. H. Rasmussen, and M. F. Rasmussen, "SARUS: A synthetic aperture real-time ultrasound system," IEEE Trans. Ultrason., Ferroelec., Freq. Contr., vol. 60, no. 9, pp. 1838-1852, 2013.

[6] J. Jensen, C. A. Villagomez-Hoyos, M. B. Stuart, C. Ewertsen, M. B. Nielsen, and J. A. Jensen, "Fast plane wave 2-D vector flow imaging using transverse oscillation and directional beamforming," IEEE Trans. Ultrason., Ferroelec., Freq. Contr., vol. 64, no. 7, pp. 1050-1062, 2017.

[7] C. A. Villagomez-Hoyos, J. Jensen, C. Ewertsen, K. L. Hansen, M. B. Nielsen, and J. A. Jensen, "Energy based clutter filtering for vector flow imaging," in Proc. IEEE Ultrason. Symp., 2017, pp. 1-4.

[8] S. S. M. Lai, B. Y. S. Yiu, A. K. K. Poon, and A. C. H. Yu, "Design of anthropomorphic flow phantoms based on rapid prototyping of compliant vessel geometries," Ultrasound Med. Biol., vol. 39, no. 9, pp. 1654 1664, 2013.

[9] J. R. Womersley, "Oscillatory motion of a viscous liquid in a thin-walled elastic tube. I: The linear approximation for long waves," Phil. Mag., vol. 46, pp. 199-221, 1955.

[10] D. H. Evans, "Some aspects of the relationship between instantaneous volumetric blood flow and continuous wave Doppler ultrasound recordings III,' Ultrasound Med. Biol., vol. 8, pp. 617-623, 1982. 